\title{
Compact, Photoacoustic Gas Measuring System for Methane in Biogas Plants
}

\author{
Jochen Huber ${ }^{1}$, André Eberhardt ${ }^{2}$, Jürgen Wöllenstein ${ }^{1,2}$ \\ ${ }^{1}$ Fraunhofer Institute for Physical Measurement Techniques (IPM), Heidenhofstr. 8, \\ 79110 Freiburg, Germany \\ jochen.huber@ipm.fraunhofer.de \\ ${ }^{2}$ IMTEK, Department of Microsystems Engineering, Faculty of Applied Sciences, \\ University of Freiburg, Georges-Köhler-Allee 102, 79110 Freiburg, Germany
}

\begin{abstract}
We present a miniaturized photoacoustic gas measurement setup for methane in high concentrations. The system consists of an IR-source, a microphone as detection unit and a measuring chamber with gas connections. The system is able to detect methane concentrations of $0-100 \%$, as they exist for example inside the reaction chamber of biogas plants. Simulations with parameter variations are done to evaluate experimental results. Measurements and simulations show similar behaviour. Different measurement ranges have been regarded. The system presented here can be easily integrated in process environment due to its small size. The isolated setup allows to measure flammable IR-active gases.
\end{abstract}

Key words: photoacoustics, gas sensor, IR-absorption, methane, biogas

\section{Introduction}

Measuring the composition of gases in biogas plants is of central importance. For example the energy content of a biogas can be derived from the gas composition. Furthermore the state of the fermentation process inside large scale plants can be ascertained from the knowledge of the gases inside. Thereby detecting the concentration of methane $\left(\mathrm{CH}_{4}\right)$ is a main focus, because $\mathrm{CH}_{4}$ is the main component of biogas [1]. $\mathrm{CH}_{4}$ concentration of biogas is also a direct indicator for the fuel value. Because of this fact small and integrated measurement systems are needed to discover $\mathrm{CH}_{4}$ concentration in gases. Required detectable concentrations in biogases are in a wide range from $30 \%$ up to $90 \%$. Most commercially available measurement systems are based on flame ionization detection or infrared laser absorption spectroscopy [[2],[3]. These systems have large volumes and are cost-intensive [4]. The measurement system presented here is based on the principle of photoacoustics. Gas molecules absorb energy of infrared radiation and convert this energy into kinetic energy. If the radiation is modulated over time, the change in kinetic energy of the gas can be detected as a time-modulated pressure difference in a closed volume. The pressure difference is a qualitative degree for the inside gas concentration of the absorbing gas. $\mathrm{CH}_{4}$ shows strong absorption of IR radiation. Its main absorption range for $\mathrm{CH}_{4}$ is around $3.4 \mu \mathrm{m}$ wavelength. Detection unit for the pressure signal is a simple commercially available lowcost MEMS microphone. The photoacoustic system has an isolated setup, so that IR-source and flammable gas are not in direct but in optical contact. The system presented here is much cheaper than usual biogas monitoring setups and can be integrated easily in various process systems.

\section{Theory and Simulation}

IR-active molecules interact with IR-radiation by absorbing specific photons. Quantized photon energy levels cause oscillations and vibrations in molecules, when the molecule's dipole moment is shifted by the motion. This quantized absorption lines give a specific "fingerprint" to each IR-active molecule. IR radiation energy is changed to kinetic energy of the molecule. This change in kinetic energy causes a pressure difference inside a closed volume. Absorption of radiation in a medium shows exponential dependence between radiation intensity and 
absorption distance. It is described by BeerLamberts-law. The energy levels of IR radiation are not high enough to cause electronic transitions, but characteristic vibrations are excited. A transmission profile $T(\lambda)$ of an absorption spectrum in the IR region for $20 \%$ $\mathrm{CH}_{4}$ in nitrogen can be seen in Fig. 1 [7]. To get the power $\mathrm{P}_{\text {ges }}$ that is absorbed by an absorbing gas, the integral of the multiplied power profile of the infrared source $P(\lambda)$ with the absorption profile $A(\lambda)=1-T(\lambda)$ can be calculated. As infrared source, an ideal black body is assumed.

$P_{g e s}=\int_{\lambda_{1}}^{\lambda_{2}} A(\lambda) \cdot P(\lambda) d \lambda$

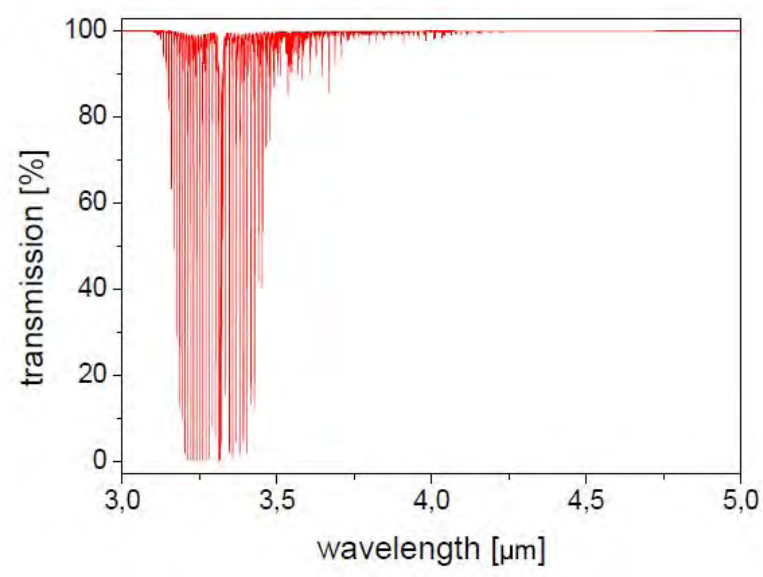

Fig. 1: Transmission profile of $20 \% \mathrm{CH}_{4}$ in nitrogen at $T=25^{\circ} \mathrm{C}, \quad p=1013 \mathrm{mbar}$ and absorption length $\Delta x=1.28 \mathrm{~cm}$ in 3-5 $\mu \mathrm{m}$ wavelength range [7].

Estimating ideal performance and neglecting all loss mechanisms (reflections, scattering, heating) all the power is absorbed in the gas. To get the energy $Q$ that is inserted in the gas volume, modulation frequency $f$ and duty cycle $v$ of the IR-source have to be regarded. We assume a rectangular function with 0.5 duty cycle, to have $50 \%$ of the time power entering and a frequency of $10 \mathrm{~Hz}$ :

$Q=\frac{P_{g e s} \cdot v}{f}$

Under the assumption of an ideal gas, the heat capacitance for an ideal gas $\mathrm{C}_{\mathrm{V}}$ is constant. With this parameter the temperature difference $\Delta \mathrm{T}$ for each light cycle can be calculated.

$\Delta T=\frac{Q}{C_{V}}$

The ideal gas equation gives the opportunity to calculate directly a pressure difference $\Delta p$ :
$\Delta p=\frac{n \cdot R \cdot T}{V}$

In the photoacoustic method, the absorbed intensity which is proportional to the amplitude of the wave, is measured directly [5]. To get values for absorption strength at a specific wavelength we used data from HITRAN [7] to get the absorption lines with broadening and intensities. Data is imported and integration is done numerically over the wavelength region. A simulation profile of the values for pressure differences in dependence to gas concentration can be seen in Fig. 2: Simulation of the pressure signal inside the measuring chamber for different concentrations of $\mathrm{CH}_{4}$ in nitrogen.. In various simulations, absorption length as parameter has been varied. Various absorption lengths take into account that reflections inside the chamber can lead to an effectively longer absorption path than the single length of the chamber.

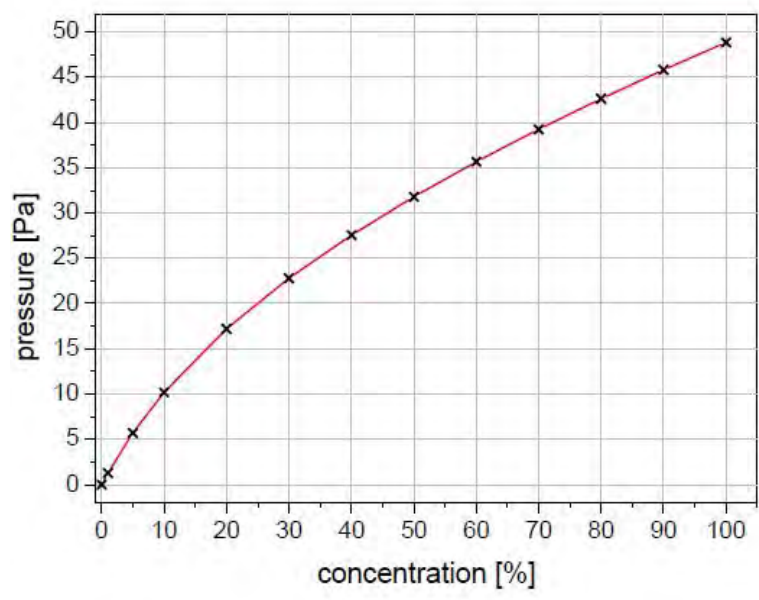

Fig. 2: Simulation of the pressure signal inside the measuring chamber for different concentrations of $\mathrm{CH}_{4}$ in nitrogen. Absorption length is assumed as $1.28 \mathrm{~cm}$. Frequency is $10 \mathrm{~Hz}$ with a rectangular signal with $50 \%$ duty cycle.

\section{Measurement Setup}

The measurement system consists of a measuring cell with a top plate, a broadband IRsource, an IR-transparent window and a MEMS microphone. The measuring cell is made of aluminum. Volume of the chamber $(\mathrm{V}=$ $170 \mathrm{~mm}^{3}$ ) should be small because the pressure signal is inversely proportional to the chamber volume [6]. The chamber has an opening to fix an IR-transparent sapphire glass window (diameter $=12.7 \mathrm{~mm}$, thickness = $3 \mathrm{~mm}$ ) that is sealed by pressing a polymer ring between glass and chamber. The window is pressed by an aluminum cover plate with 
openings for microphone and IR-window. The microphone is a commercially available MEMSmicrophone (SMM310, Infineon). Gas inlet and outlet are installed for measurements as it is shown in Fig. 3.

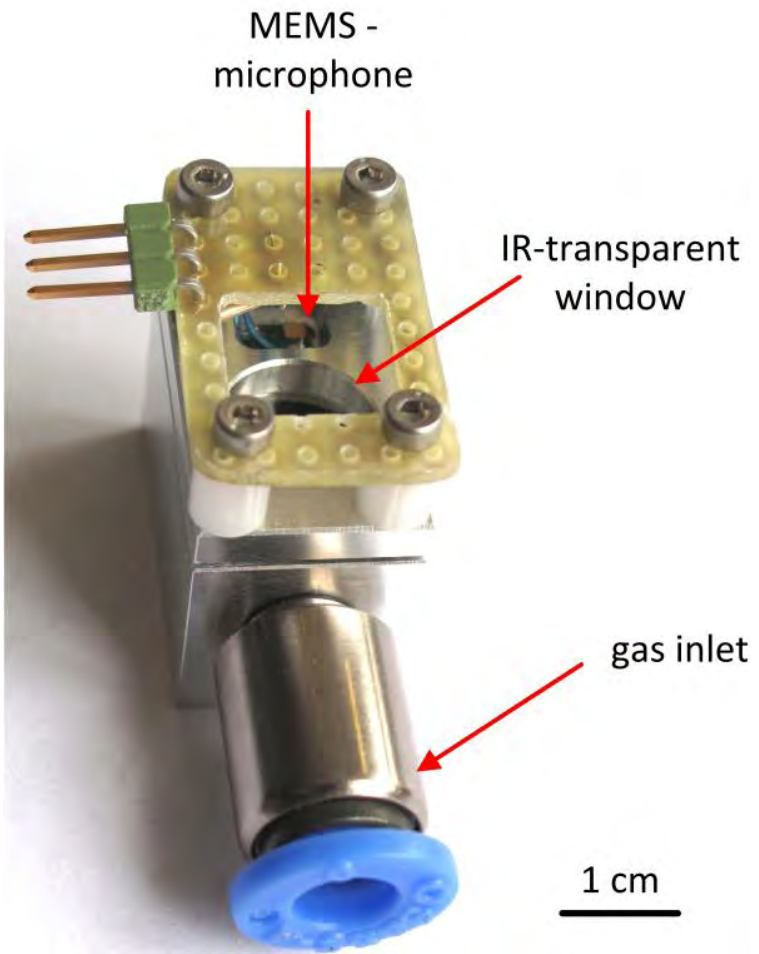

Fig. 3: Measuring chamber setup with mounted top plate, IR-transparent window, MEMS-microphone and gas peripheral components.

The microphone signal has a high noise and has to be filtered, amplified and digitized to evaluate a comparable value. Data analysis is done close to the detector to have less electrical interferences and a better signal-tonoise ratio [8]. Filtering is realized digitally in a PSoC5 microcontroller. The filter routine is a digitally realized so called Lock-in-amplifier. A Lock-in-amplifier is an amplifying, highly frequency-selective, narrow bandpass filter to analyze alternating signals. Output signal is a digital value corresponding to the amplitude of the alternating signal. The reference signal that is needed for the Lock-in-amplifier with the same frequency as the measurement signal is taken from the modulation actuation of the IRsource, which is also realized with the PSoC5 microcontroller. As IR-source a broadband thermal emitter (IR-66, Hawkeye) is used. In Fig. 4 a measurement over the whole measurement range is shown. The measured values are allometrically fitted. Measurement values are averaged of the result of 13 measurements. The system consists of a single chamber with two peripheral connectors. To measure an acoustic signal with the microphone, the volume has to be hermetically sealed. Otherwise the pressure gradient would be buffered and compensated by the big volume around the chamber. To seal the chamber after inserting a probe gas, valves have been mounted directly in front and behind the measuring chamber.

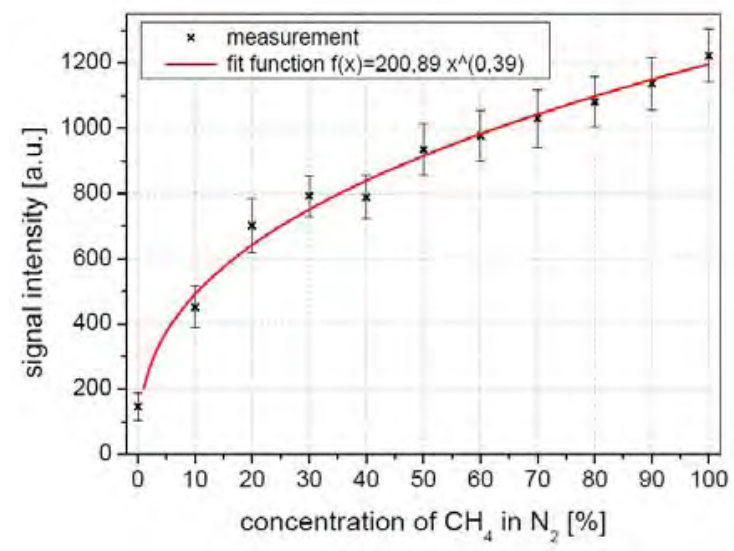

Fig. 4: Measurement between $0-100 \% \mathrm{CH}_{4}$ in $\mathrm{N}_{2}$. Measurement values are averaged with the results of 13 measurements. Result is fitted with an allometric fit function. On the $y$-axis the analyzed digital value without a unit is drawn

\section{Experimental Results and Discussion}

Performed measurements show the working principle and show also the possibility to use the system in the expected environment. To realize a real time measurement system, electronic controlled valves or a second and sealed reference chamber can be used. First measurement was done to cover the measurement range from $0-100 \%$ of $\mathrm{CH}_{4}$ in nitrogen. It can be seen that there is no saturation effect at a specific concentration. This is probably because of the small volume of the chamber. The signal is reproducible, but the noise does not allow a higher resolution than $5 \%$ steps of concentration over the whole measurement range. The exponential fit curve shows high resolution in the region from $0 \%$ to $10 \% \mathrm{CH}_{4}$. An optimization of the optical path length and the chamber volume can achieve a better resolution for defined detection ranges. Fig. 5 shows comparison between measurement and simulation. Measurement results are digitized values without any unit after digital filtering with the microcontroller. Simulation results are pressure values in $\mathrm{Pa}$. To compare measurement and simulation curves, a reference calculation is done. As reference fixed-point maximum signal at $100 \% \mathrm{CH}_{4}$ is taken. At this gas concentration absolute maximum value of the signal is reached. All other values are represented as percentage 
values of this maximum. The change of the signals with reference to the maximum signal shows same progress for both curves. Calculation and experimental results fit well. For the simulation curve in Fig. 5 double absorption path is taken. This value for absorption path gives best result for comparison. That means the light passes two times the cell in average. Simulations give the possibility to optimize geometrical parameters of the setup for different measurement ranges

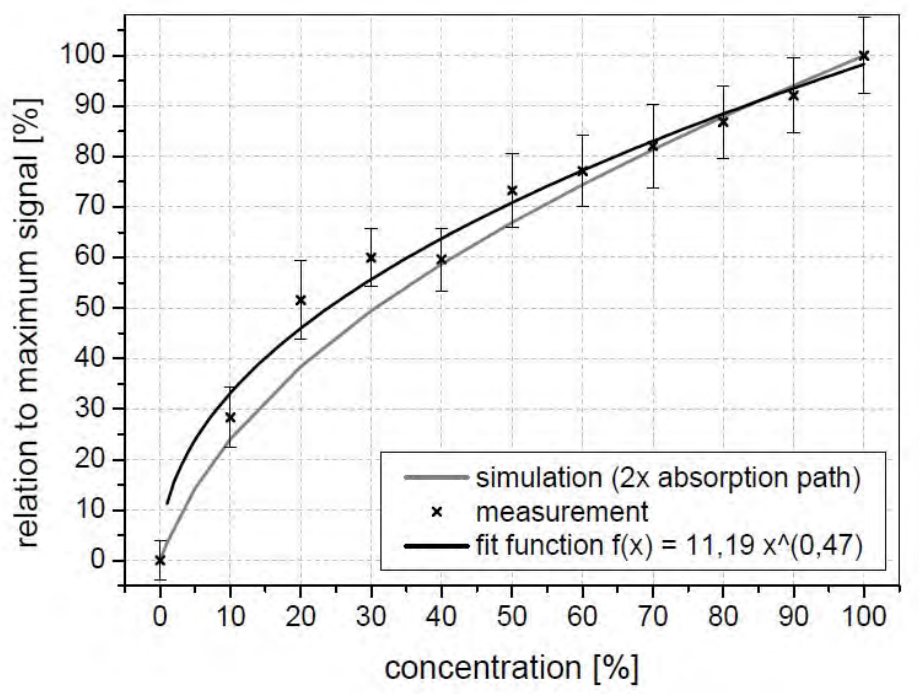

Fig. 5: Comparison of measured and calculated signal. Both curves are referenced to the fixed-point at maximum value at $100 \% \mathrm{CH}_{4}$. Measurement values are fitted allometrically.

\section{Conclusion and Outlook}

The system presented here can be used as a small, integrated setup to detect high $\mathrm{CH}_{4}$ concentrations as they exist in biogas plants. Small size of the setup allows easy integration. Components are commercially available and reduce costs of the system. A big measurement range $(0-100 \%)$ can be regarded. Measurement principle is a physical effect and represents directly the presence of the detectable gas. Flammable gases like $\mathrm{CH}_{4}$ can be measured well with presented setup because radiation source and gas are physically separated but optically connected. Thus the IR-radiation source cannot appear as ignition source. Electronic data conversion and analysis is implemented in a PSoC5 microcontroller. In future work a second chamber, working as reference chamber can be integrated. That gives the possibility to measure in real-time. Furthermore no valves are needed which reduces complexity of the system. Full Integration of the complete system in a sensor housing is aspired to have a small, cheap and highly integrated sensor-system. Optimization due to chamber volume and aspired measurement range can be done with simulations.

\section{References}

[1] Kaltschmitt M., Hartmann H., Hofbauer H., Energie aus Biomasse (2. Auflage), 2009, Springer-Verlag, Heidelberg

[2] Martin, P.A., Near-infrared diode laser spectroscopy in chemical process and environmental air monitoring, Chem. Soc. Rev., 2002, 31, 201-210

[3] Nielsen H.B., Angelidaki I., Strategies for optimizing recovery of the biogas process following ammonia inhibition, Bioresource Technology 99, 2008 7995-8001

[4] Schmid T., Photoacoustic spectroscopy for process analysis. Analytical and Bioanalytical Chemistry 384 (2006), 1071-1086.

[5] Kauppinen J, et al., High sensitivity in gas analysis with photoacoustic detection. Microchemical Journal 76, 2004, 151-159

[6] Firebaugh S.L., Jensen K.F., Schmidt M.A., Miniaturization and Integration of Photoacoustic Detection with a Microfabricated Chemical Reactor System. Journal of Microelectrochemical Systems Vol. 10, 2001

[7] HITRAN spectral database 2008, www.cfa.harvard.edu/HITRAN.

[8] Schrüfer E., Elektrische Messtechnik, 7. Auflage, 2001, Carl Hanser Verlag, München 